\title{
Nuevo asentamiento de época romana en Navarra
}

\author{
María Jesús Peréx Agorreta * \\ Merteded UnZU URMeneta **
}

La última campaña de excavación llevada a cabo en Espinal (Navarra), en septiembre de 1988, se ha centrado en el poblado al que corresponde la necrópolis de incineración descubierta en 1986, y ya excavada (fig. 1).

Comenzamos los trabajos teniendo como referencia los restos de un muro aparecido al final de la campaña de 1987 (dedicada, primordialmente, a la necrópolis), cuya orientación aproximada es Noroeste-Sudeste. A ambos lados de dicho muro pudimos recuperar gran cantidad de cerámica de época romana, tanto T.S.H. como de cocina y común. Por lo tanto, las catas (de 2,5 por 2,5) se planificaron en función de dicho muro, abriéndose un total de veintitrés.

Tal como sucedía en la zona de la necrópolis (distante unos $250 \mathrm{~m}$. del poblado) los restos constructivos y cerámicos se encontraban a muy escasa profundida, entre los 30 y $40 \mathrm{cms}$.

De forma paralela y perpendicular al muro mencionado más arriba, fueron apareciendo los restos de, al menos, dos habitaciones (fig. 2).

Aunque empleamos el término "muro", nos encontramos, en realidad, con una sola hilada de piedras, colocada sobre el terreno virgen, constituído por un tipo de roca grisácea, muy quebradiza, que recibe el nombre de tufa. Pensamos que sobre esta primera hilada se asentaria un zócalo, de una altura difícil de precisar por el momento, sobre el que se apoyarian los muros de madera y tapial.

El arranque de los zócalos tiene una anchura de $0,60 \mathrm{~m}$. y está formado por piedras calizas y por la llamada piedra de Arrieta, similar a una

* Departamento de Prehistoria e H. Antigua (UNED-Madrid).

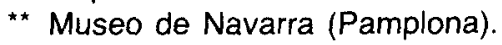




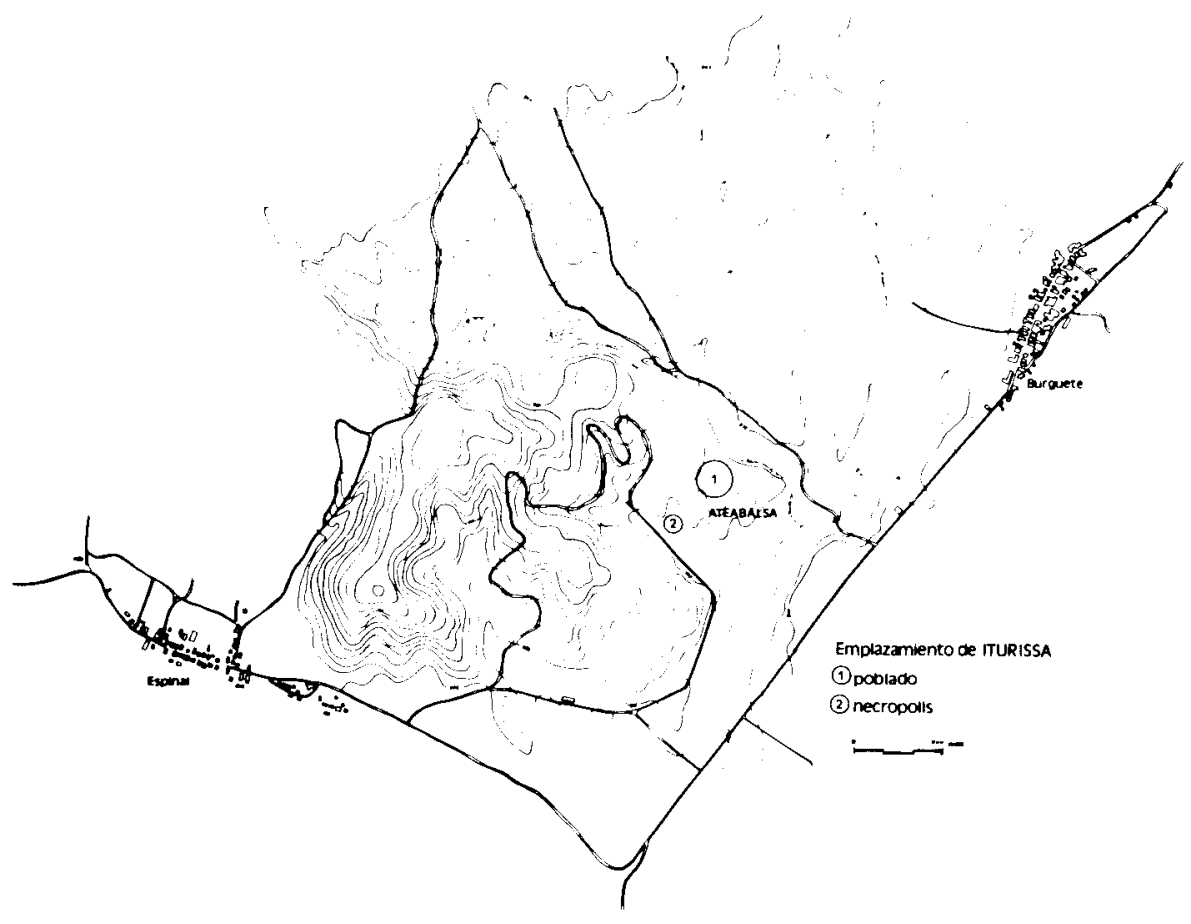

Fig. 1. Localización de la zona excavada. 


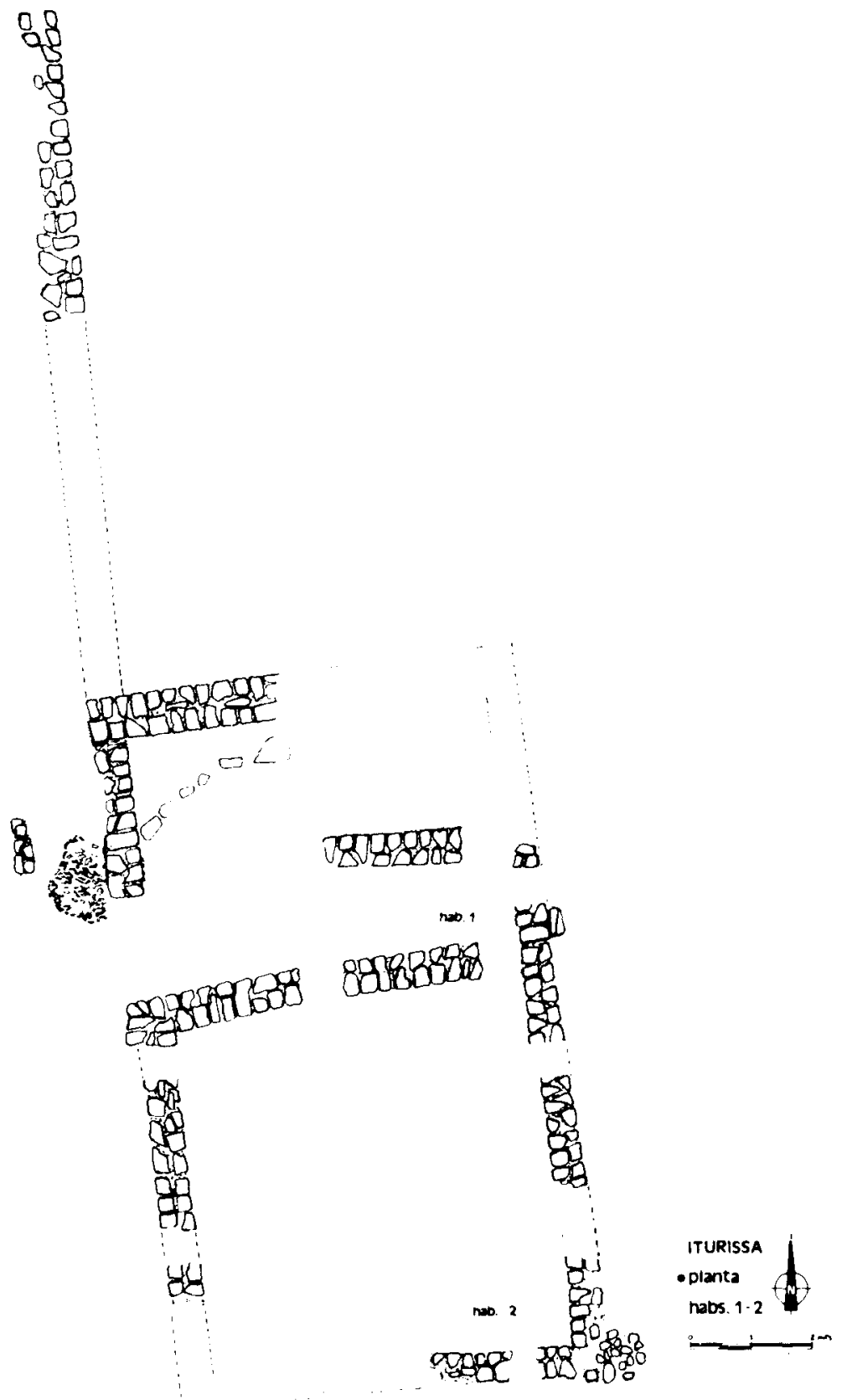

Fig. 2. Plano de las habitaciones excavadas. 
pizarra rojiza, cuya cantera se encuentra a unos ocho kilómetros del yacimiento (en el término de Arrieta, de ahi su nombre). Se colocaban ambas entre la tufa propia del terreno para dar consistencia al muro, ya que este tipo de roca es muy trágil.

Suponemos que el material más utilizado sería la madera, dada la escasez de piedra en las proximidades, mientras que el arbolado antes, como ahora, debió ser muy abundante. Corrobora esta opinión el hallazgo, en toda la zona excavada, de numerorísismos clavos (de cabeza circular y de sección del vástago cuadrada). A excepción de la piedra y de un solo ladrillo (de 16 por $16 \mathrm{~cm}$.) no se han encontrado restos de otros materiales constructivos, por lo que pensamos que las cubiertas de las habitaciones pudieron ser de tablillas tal como han perdurado hasta nuestros días, y con carácter excepcional por lo que a Navarra se refiere, en esta zona concreta del Pirineo Occidental.

El hecho de que la zona en la que se asienta el yacimiento haya sido cultivada durante varios años consecutivos, tras roturarse a finales de los años 50 para convertir en parcelas estos terrenos comunales, hace que los hallazgos se concentren en torno a un haya, de enormes proporciones (a la que se le han calculado unos doscientos años de antigüedad), cuyas raíces estorbaban los trabajos del arado. No obstante, junto a ella, y en las lindes de separación de parcelas, aparecen gran cantidad de piedras, alli depositadas para despejar las zonas de cultivo, de la misma factura que las halladas formando los muros.

Como ya hemos dicho más arriba, los hallazgos principales se concretan en la recuperación de gran cantidad de fragmentos de cerámica de cocina, común y T.S.H. La característica más destacable de todos ellos es su mal estado de conservación, que impide su normal tratamiento como sería el lavado previo. Aunque aparecen diseminados por toda la zona excavada, la mayor concentración se halla situada en torno a uno de los muros (cuadriculas 2, 6, 7 y 12) (ver fotografia de la fig. 3). Aquí nos encontramos con un estrato de unos $15 \mathrm{~cm}$. de potencia en el que aparecen mezclados fragmentos de grandes vasijas de cerámica tosca, de cocina, junto a otros de cerámica común, de color anaranjado claro, y diversas formas de T.S.H., tanto lisas como decoradas, siendo las más frecuentes la 25, 35, 37 a y $37 \mathrm{~b}$; y también algún fragmento de sudgálica (forma 29 de Montans, de época flavia).

La cerámica de cocina corresponde al tipo de borde horizontal, cuerpo ovoide, pasta negra (cocción reductora) y superficie peinada, idónea para ser utilizada sobre el fuego. De la cerámica común, de color anaranjado, no podemos precisar todavía sus formas. 


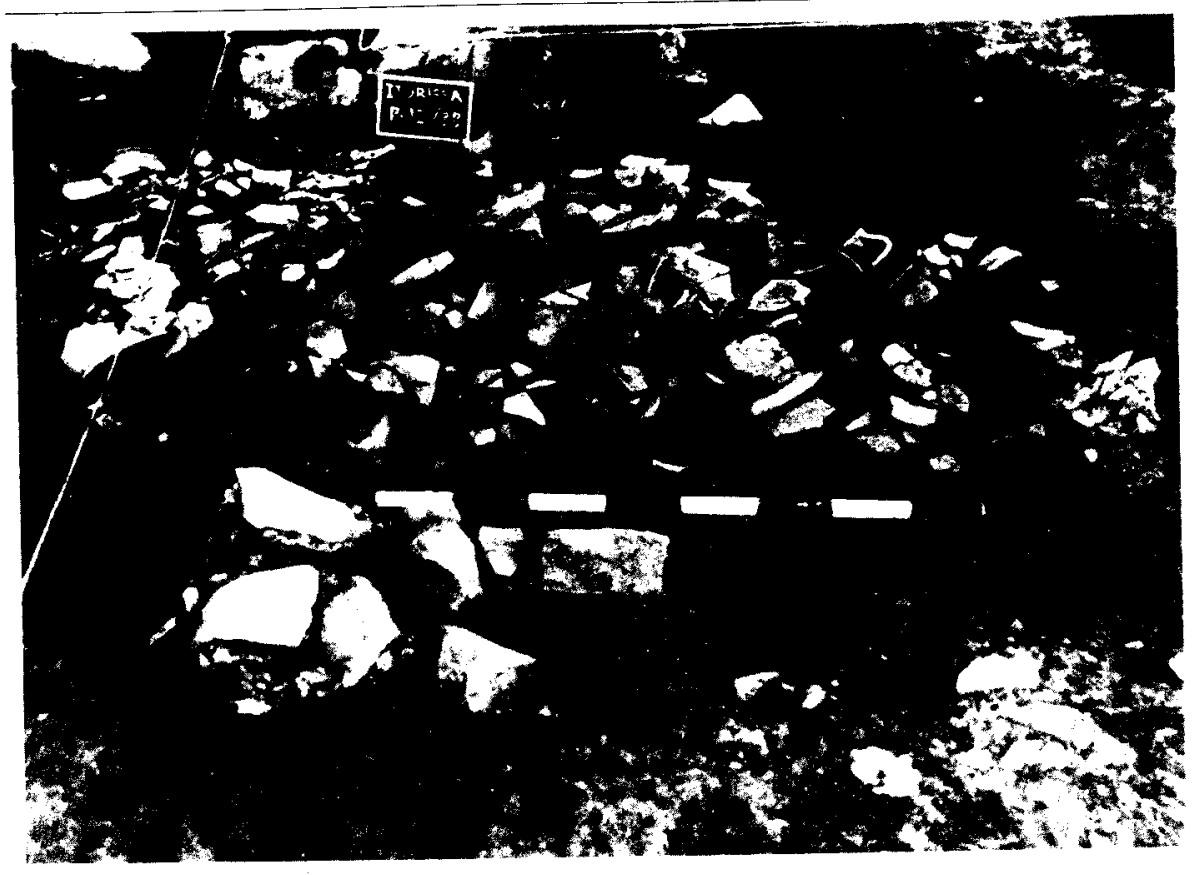

Fig. 3. Lugar de mayor concentración de materiales.

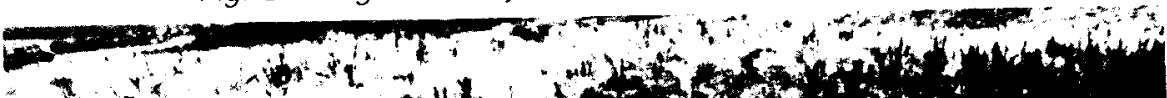

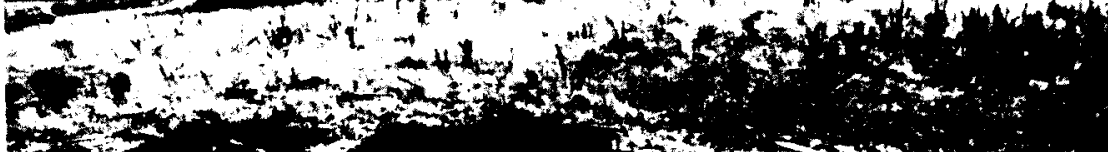

(5)

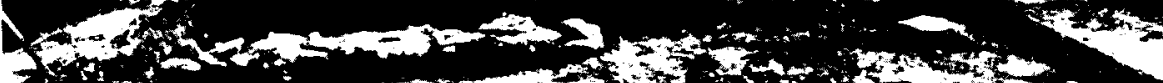

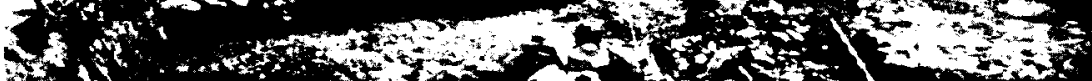

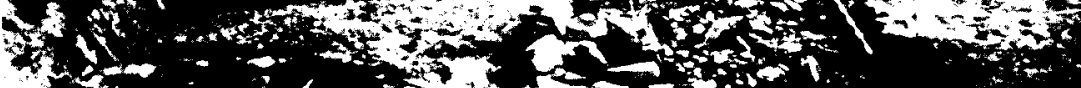

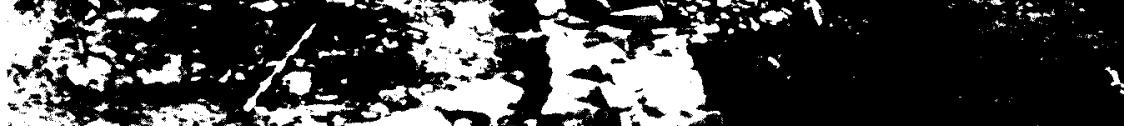

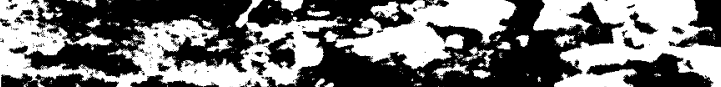

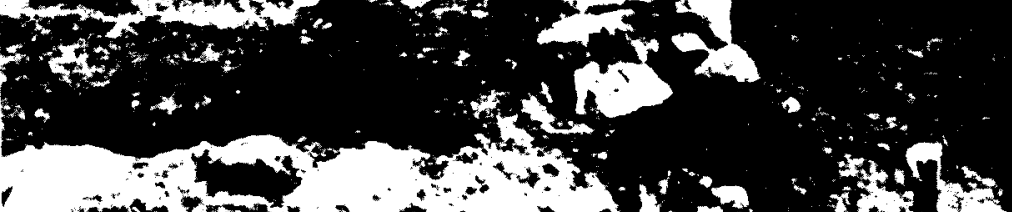

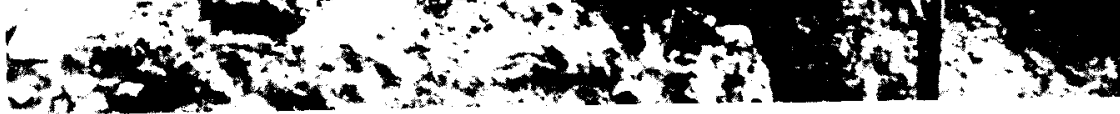
Fig. 4. Habitación número 1. 
Como indicamos anteriormente, se han localizado dos habitaciones. La primera (habitación $n .{ }^{\circ}$ 1) (ver fotografía fig. 4) mide unos $24 \mathrm{~m}^{2}$, y en ella se concentran el mayor número de hallazgos cerámicos y también numerosos fragmentos de vidrio, siendo las asitas (cuatro) las piezas mejor conservadas, además del cuello, el borde y el arranque del asa de un jarrito de vidrio de mayor tamaño. También recuperamos aquí una cuenta de collar de pasta vítrea.

Es de destacar la existencia, bajo el muro Oeste de esta habitación (ver fotografía fig. 5, a la izquierda), de gran cantidad de fragmentos cerámicos, tanto de cerámica común como de T.S.H., que forman una capa sobre la que se asienta. La explicación puede estar en la utilización de vasijas fuera de uso como aislante de la humedad, muy abundante en la zona. Debido a que en futuras campañas esperamos ampliar la zona excavada, para poder obtener una mejor visión del conjunto del poblado, no hemos querido levantar dicho muro, de tal forma que no podemos precisar la cronologia de la cerámica asi depositada.

En el testigo situado al Este de las cuadrículas 2 y 7 (correspondientes a la habitación $\mathrm{n} .^{\circ} 1$ ), se aprecia un estrato, de unos 12 a $15 \mathrm{~cm}$. de espesor y color anaranjado, que podría corresponder al derrumbe de un tabique de ladrillo (del que, como ya hemos dicho, solo se ha recuperado un ejemplar).

La habitación $n .^{\circ} 2$, que pide unos $36 \mathrm{~m}^{2}$, ha proporcionado muy pocos restos. Situada al Sudesde de la anterior, suponemos que dicha escasez se debe a que, dada la poca profundidad en que se encontraron los muros, los sucesivos trabajos de labranza debieron dispersar los materiales.

En cuanto a la posible adscripción a este yacimiento de alguna de las localidades mencionadas por Ptolomeo (II, 6, 67) como pertenecientes a los vascones, nos inclinamos por su identificación con Iturissa. Esta localidad aparece también en los Itinerarios (It. Ant. 445,6 y An. Rav. IV 43 $311,14)$ como mansio en la vía de Astorga a Burdeos a su paso por el Pirineo Occidental. Los materiales recuperados hasta el momento pertenecen a los siglos I y II d.C. (ver Bibliografía al final de este trabajo), y suponen el asentamiento más septentrional del territorio vascón en época romana (fig, 6). 


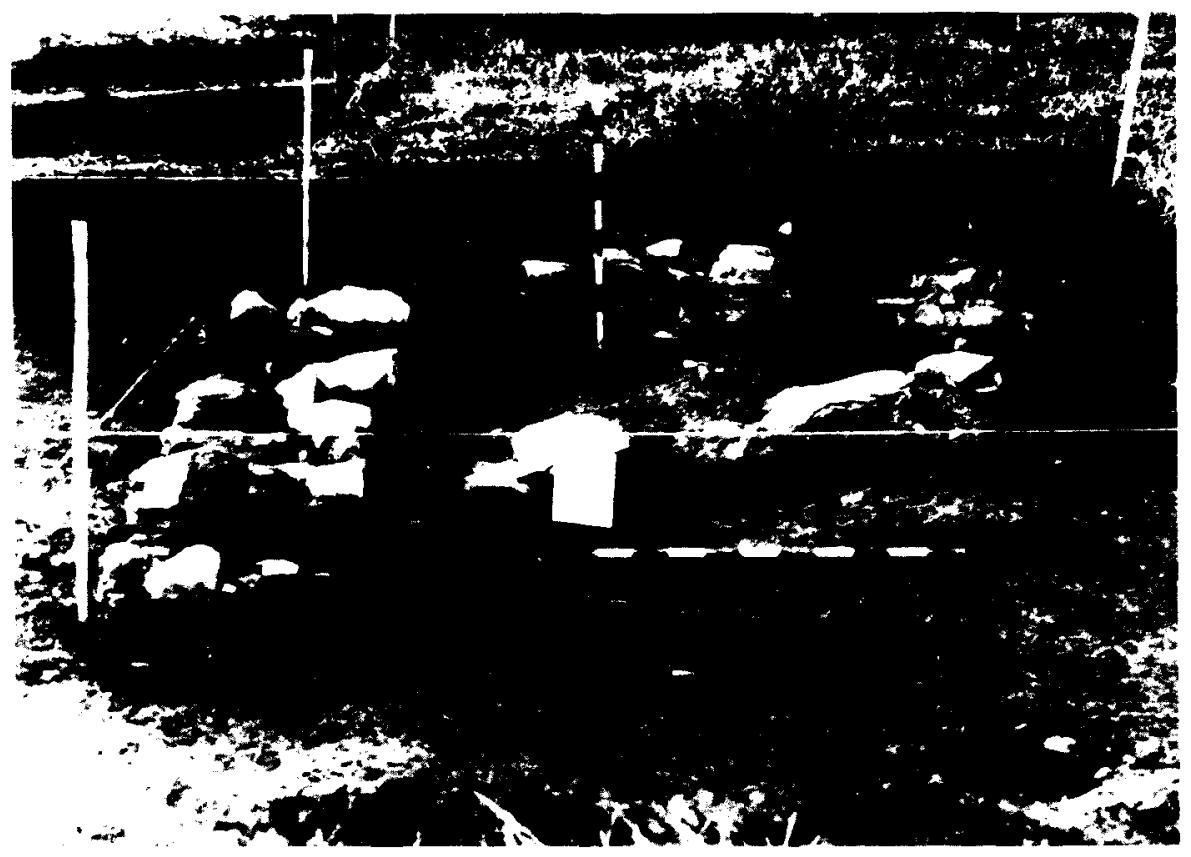

Fig. 5. Detalle de los restos cerámicos situados bajo el muro de la izquierda. 


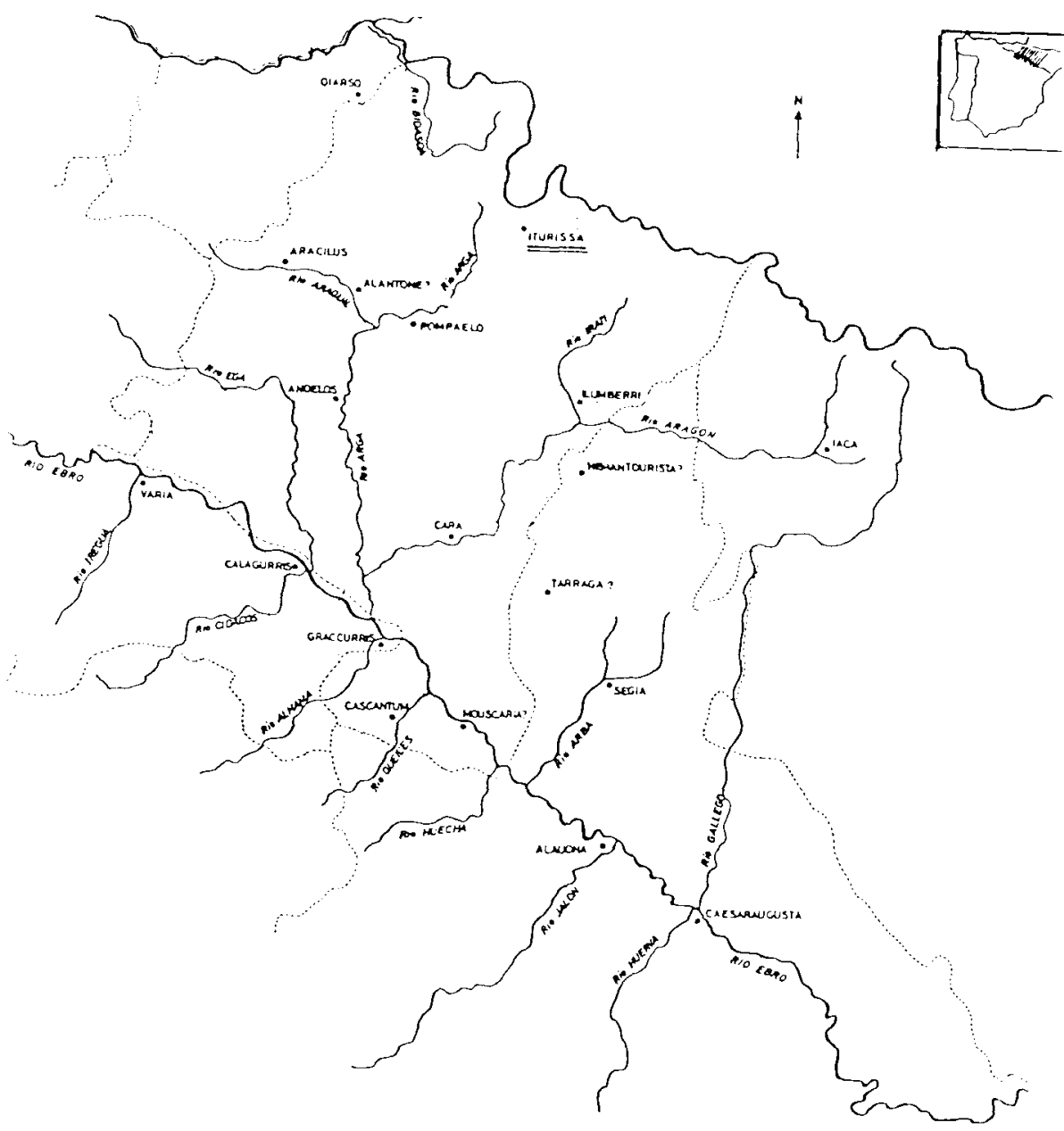

Fig. 6. Localización de Iturissa. 


\section{BIBLIOGRAFÍA}

AltADILL, J., 1928: "Geografía histórica de Navarra. Los despoblados", B.C.M.N. 52, 1922, págs. 262-264, y en "De re geographico-histórica. Vías y vestigios romanos en Navarra", Homenaje a D. Carmelo Echegaray, San Sebastián, pág. 40.

Peréx, M. J., 1986: Los vascones, Pamplona, págs. 172-179.

PERÉX, M. J., 1987: "Notas sobre la calzada romana entre Pompaelo e Iturissa (Navarra)», XVIII C.A.N. (Canarias, 1985), págs. 805-811.

PERÉX, M. J. y UNZU, M., 1987: "Notas sobre la posible localización de Iturissa (Espinal, Navarra)", Primer Congreso General de Historia de Navarra, P.V., Anejo 7, 1987, págs. 553-562.

PERÉX, M. J. y UNZU, M., 1987: “Necrópolis romana de incineración. Espinal, Navarra", Revista de Arqueología 73, 1987, págs. 58 y 59.

PERÉX, M. J. y UnzU, M., 1987: «Emplazamiènto de Iturissa, mansio en la via de Astorga a Burdeos", Simposio sobre la red viaria en la Hispania Romana, Tarazona, (en prensa).

Pérez de Villarreal, V., 1981: «Minetako zokoa. El lugar de las minas. (Baztán-Navarra)», C.E.E.N. 37, pág. 165.

SAYAS, J. J., 1984: “El poblamiento romano en el área de los Vascones", Veleia 1, págs. 289-310.

SAYAS, J. J. y PERÉX, M. J., 1987: «La red viaria de época romana en Navarra", Primer Congreso de Historia de Navarra, P. V., Anejo 7, págs. 581-608. 